\title{
Editorial: Optics and Ecophysiology of Coral Reef Organisms
}

\author{
Daniel Wangpraseurt ${ }^{1,2 *}$, Anthony W. D. Larkum ${ }^{3}$, Christine Ferrier-Pagès ${ }^{4}$, Anya Salih ${ }^{5}$, \\ Mark E. Warner ${ }^{6}$, Zvy Dubinsky $^{7}$ and Michael Küh/ ${ }^{8}$
}

${ }^{1}$ Department of Chemistry, University of Cambridge, Cambridge, United Kingdom, ${ }^{2}$ Marine Biology Research Division, Scripps Institution of Oceanography, University of California, San Diego, La Jolla, CA, United States, ${ }^{3}$ Climate Change Cluster, University of Technology Sydney, Ultimo, NSW, Australia, ${ }^{4}$ Ecophysiology Team, Scientific Centre of Monaco, Monaco, Monaco, ${ }^{5}$ School of Science and Health, Hawkesbury Institute for the Environment, Western Sydney University, Penrith, NSW, Australia, ${ }^{6}$ School of Marine Science and Policy, University of Delaware, Newark, DE, United States, ${ }^{7}$ The Mina and Everard Goodman Faculty of Life Sciences, Bar-llan University, Ramat Gan, Israel, ${ }^{8}$ Department of Biology, Marine Biological Section, University of Copenhagen, Copenhagen, Denmark

Keywords: photosynthesis, coral reefs, photobiology, optics, coral bleaching

\section{Editorial on the Research Topic}

\section{Optics and Ecophysiology of Coral Reef Organisms}

\section{OPEN ACCESS}

Edited and reviewed by: Raquel Peixoto,

Federal University of Rio de Janeiro, Brazil

*Correspondence: Daniel Wangpraseurt dw527@cam.ac.uk

Specialty section: This article was submitted to Coral Reef Research, a section of the journal Frontiers in Marine Science

Received: 23 September 2019 Accepted: 14 November 2019 Published: 03 December 2019

Citation:

Wangpraseurt D, Larkum AWD, Ferrier-Pagès C, Salih A, Warner ME,

Dubinsky $Z$ and Kühl M (2019) Editorial: Optics and Ecophysiology of Coral Reef Organisms.

Front. Mar. Sci. 6:742.

doi: 10.3389/fmars.2019.00742
Light is arguably the most important factor driving the evolutionary success of coral reefs (Falkowski et al., 1990). Solar energy is the prime force underlying the symbiotic interaction between phototrophic dinoflagellates of the family Symbiodiniaceae and the heterotrophic coral animal host. Light is also the major energy source for other key coral reef organisms such as crustose coralline algae, sponges, foraminifera, and epi- and endolithic phototrophic biofilms. However, the physical interaction between light and reef organisms is often treated in insufficient detail. For instance, the spectral and directional characteristics of sunlight, as well as the high temporal and spatial variability, can strongly modulate the photosynthetic response of reef organisms, yet are often ignored (Roth, 2014). Likewise, the optical properties of reef organisms, such as the scattering properties of their photosynthetic tissues, the presence of fluorescent pigments and the structural properties of their calcium carbonate skeleton can strongly modulate light absorption and photosynthesis (Salih et al., 2000; Wangpraseurt et al., 2019a). The present Research Topic is a compilation of 14 articles that aim to improve our understanding of how light affects the ecophysiology of coral reef organisms. The topic is divided into (i) articles that discuss the interaction between light and coral reef organisms and (ii) articles that showcase new technologies enabling an improved understanding of the optics of coral reefs.

The articles by Wangpraseurt et al., Swain et al., and Scheufen et al. discuss relationships between optical properties, photophysiology and bleaching susceptibility of shallow water corals. Wangpraseurt et al., used optical microsensors to study the in vivo light microhabitat inside coral tissues during bleaching. It is shown how symbiont loss can lead to an exponential acceleration of the internal light field, suggesting an enhanced contribution of coral scattering for the remaining symbionts. Such an optical feedback mechanism was originally proposed by Enriquez et al. (2005), and has now been confirmed by direct measurements of the light microclimate in corals. However, Wangpraseurt et al. found such accelerating light exposure to be species dependent-it was pronounced in thin-tissued Pocillopora damicornis corals, while thick-tissued Favites sp. showed a more linear increase in irradiance as a function of symbiont loss. Likewise, Swain et al. provide further evidence for the optical feedback loop using low coherence enhanced backscattering spectroscopy and an optical model to understand coral skeletal light propagation. They show that bleaching susceptibility was strongly related to the scattering coefficient of the top $200 \mu \mathrm{m}$ of the skeletons. A fractal skeletal growth model suggests that corals that grow faster typically have a lower mass-fractal dimension, denser skeletons with lower reduced scattering coefficients and 
higher bleaching susceptibility. Scheufen et al. compared optical traits and structural characteristics of a range of corals, which differ in skeleton morphology, tissue thickness, coral pigmentation, symbiont type and density. The authors show that light absorption capacity was related to the architectural complexity of the coral skeletons, with chalice-type skeletons achieving higher light absorption capacity than meandroid-type skeletons. Together, these three articles highlight the complexity of light-matter interaction in corals, which is modulated by coral tissue properties, skeleton fractal length, and architectural complexity along with the photosynthetic properties of the coral photosymbionts.

Coral optical properties are also strongly affected by spatial and temporal variability. The articles by Einbinder et al., Ezzat et al., and Nitschke et al. focus on photoacclimation and photoadaptation of Symbiodinum over time and space. Mesophotic coral reefs have recently been studied with great interest due to their potential to serve as refugia during climate change, but limited knowledge exists on the photosynthetic properties of Symbiodiniaceae from such water depths. Einbinder et al. found that mesophotic Stylophora pistillata performed below their photosynthetic compensation point and exhibited a distinct light harvesting antenna organization in their photosymbionts, where Symbiodiniaceae developed a cooperative system for excitation energy transfer between photosynthetic units that maximizes light utilization. Ezzat et al. also found that shallow water S. pistillata associate with Symbiodinium clade A, while mesophotic $S$. pistillata preferably associate with Symbiodinium clade $\mathrm{C}$ that is more efficient at fixing carbon under low light conditions. Thus, both a change in the antenna organization of Symbiodinium and differences in symbiont type are successful adaptations to low light environments. Nitschke et al., characterized the PSII photophysiology of corals from shallow reef environments in spring vs. summer. Despite significant seasonal differences in photoacclimation, the thermal tolerance of coral species did overall not shift between seasons.

Green fluorescent protein (GFP)-like host pigment are produced by many corals but the function of GFPs is still disputed with studies showing both a role in photoprotection (Salih et al., 2000), light enhancement, and tissue heating (Lyndby et al., 2016; Smith et al., 2017; Wangpraseurt et al., 2019a). Quick et al. studied the trade-offs associated with photoprotective GFP expression as a driver for coral polymorphism. Excess blue light excitation was less detrimental to highly fluorescent color morphs compared to low fluorescent morphs. Under low irradiance, however, growth rates were lowered in the highly fluorescent morph, highlighting that host pigments synthesis involves energetically costly protein expression. Color polymorphism caused by photoprotective GFPs may thus be a product of balancing selection, in which high pigment content may be beneficial at the upper and detrimental at the lower end of the depth distribution range of symbiotic corals (Quick et al.). This article adds to the apparently multiplex roles that fluorescent host pigments play for coral fitness, which might not always be beneficial.

The light depended physiological activity of corals also implies variations in the local physico-chemical microhabitat, with pronounced diel variations in $\mathrm{O}_{2}$ and $\mathrm{pH}$. Silveira et al. investigated the role of diel changes in the bacterial community within the momentum boundary layer (MBL) of corals. They found that dominant taxa and functions in the MBL community were stable suggesting that water flow overrules the effects of coral physiology on the MBL bacterial community. Another article in this ebook investigated the role of light scattering in coralline algae. Vasquez-Elizondo and Enríquez studied the role of the skeleton in enhancing thallus light absorption efficiency of red coralline algae. Thicker rhodolith thalli were the most efficient light collectors exhibiting high pigment absorption efficiency and areal photosynthesis rates. This study demonstrates the utility of measuring optical traits to investigate differences in the competitive abilities, abundances, and niche distribution among algal species and/or growthforms, and further suggests that skeletal scattering is an important light enhancing mechanism in other reef organisms besides corals.

The second part of the ebook deals with new optical techniques related to the characterization of inherent optical properties, chlorophyll $a$ and GFP fluorescence. Jacques et al. describe a simple low-cost approach to characterize the bulk absorption and scattering properties of living corals via spatially resolved diffuse reflectance measurements. Mazel describes a new method to quantify the contribution of fluorescence to optical signatures under ambient illumination conditions and argues that fluorescence has an important visual function in the reef environment. Zweifler et al. developed a fluorescencebased technique to acquire daytime photographs enabling identification of coral recruits. The authors envisage that their technique will provide an easy-to-use photographic system that can contribute significantly to the toolkit for coral reef monitoring and management.

Variable chlorophyll a fluorescence is a key tool in characterizing coral health and photophysiological characteristics of corals. However, since the spatial origin of PSII chlorophyll fluorescence in coral tissues is uncertain, such signals give limited information on depth-integrated photosynthetic performance of the whole tissue (Wangpraseurt et al., 2019b). Szabó et al. developed a new approach to assess dynamics of photosystem II and I in whole coral tissues based on redox kinetics of the primary electron donor in Photosystem I (P700) and chlorophyll fluorescence kinetics (Fast-Repetition Rate fluorometry, FRRf). The authors show that P700 kinetics measurements can be used as a measure for the integrity of PSII-PSI electron transfer dynamics in corals. Lastly, Hoadley and Warner developed, a low-cost multicolor chlorophyll a fluorometer using an open source hardware development platforms (Arduino and Bitscope) to construct a multispectral excitation system. This may inspire further developments of highly customized low-cost instrumentation for coral photophysiology.

In summary, the contributions in this ebook highlight the fundamental role of the interaction between light and reef organisms and its implication for coral ecophysiology, bleaching susceptibility, photosynthetic efficiency, and other 
key ecological functions on coral reefs. It also shows that we still need a better mechanistic understanding of fundamental light-matter interactions in reef organisms, which relies on the development and application of novel techniques and experimental approaches for monitoring optical properties, light fields, and photobiological processes in reef organisms in their natural environment.

\section{AUTHOR CONTRIBUTIONS}

The editorial was written by DW, MK, CF-P, and AL with contributions from all remaining authors.

\section{REFERENCES}

Enriquez, S., Mendez, E. R., and Iglesias-Prieto, R. (2005). Multiple scattering on coral skeletons enhances light absorption by symbiotic algae. Limnol. Oceanogr. 50, 1025-1032. doi: 10.4319/lo.2005.50.4.1025

Falkowski, P. G., Jokiel, P. L., and Kinzie, R. (1990). Irradiance and corals. Ecosyst. World 25, 89-107.

Lyndby, N. H., Kühl, M., and Wangpraseurt, D. (2016). Heat generation and light scattering of green fluorescent protein-like pigments in coral tissue. Sci. Rep. 6:26599. doi: 10.1038/srep26599

Roth, M. S. (2014). The engine of the reef: photobiology of the coral-algal symbiosis. Front. Microbiol. 5:422. doi: 10.3389/fmicb.2014.00422

Salih, A., Larkum, A., Cox, G., Kühl, M., and Hoegh-Guldberg, O. (2000). Fluorescent pigments in corals are photoprotective. Nature 408, 850-853. doi: $10.1038 / 35048564$

Smith, E. G., D’angelo, C., Sharon, Y., Tchernov, D., and Wiedenmann, J. (2017). Acclimatization of symbiotic corals to mesophotic light environments through wavelength transformation by fluorescent protein pigments. Proc. R. Soc. B Biol. Sci. 284, 1-9. doi: 10.1098/rspb.2017.0320

\section{FUNDING}

DW was supported by grants from the European Union's Horizon 2020 scheme. MK was supported by grants from the Independent Research Fund Denmark (DFF-1323-00065B and DFF-8021-00308B) and the Carlsberg Foundation. MW was supported by the National Science Foundation (award 1635695).

\section{ACKNOWLEDGMENTS}

We thank the Frontiers in Marine Science Editorial staff for their invitation and support in producing this Research Topic.

Wangpraseurt, D., Jacques, S., Lyndby, N., Holm, J. B., Pages, C. F., and Kühl, M. (2019a). Microscale light management and inherent optical properties of intact corals studied with optical coherence tomography. J. R. Soc. Interface 16:20180567. doi: 10.1098/rsif.2018.0567

Wangpraseurt, D., Lichtenberg, M., Jacques, S. L., Larkum, A. W., and Kühl, M. (2019b). Optical properties of corals distort variable chlorophyll fluorescence measurements. Plant Physiol. 179, 1608-1619. doi: 10.1104/pp.18.01275

Conflict of Interest: The authors declare that the research was conducted in the absence of any commercial or financial relationships that could be construed as a potential conflict of interest.

Copyright (c) 2019 Wangpraseurt, Larkum, Ferrier-Pagès, Salih, Warner, Dubinsky and Kühl. This is an open-access article distributed under the terms of the Creative Commons Attribution License (CC BY). The use, distribution or reproduction in other forums is permitted, provided the original author(s) and the copyright owner(s) are credited and that the original publication in this journal is cited, in accordance with accepted academic practice. No use, distribution or reproduction is permitted which does not comply with these terms. 